\title{
Biliary Glutathione Promotes the Mucosal Metabolism of Luminal Peroxidized Lipids by Rat Small Intestine In Vivo
}

Tak Yee Aw

Department of Physiology and Biophysics, Louisiana State University Medical Center, Shreveport, Louisiana 71130

\begin{abstract}
We previously found that exogenous GSH enhances mucosal GSH and promotes lipid hydroperoxide metabolism by rat small intestine (Aw, T. Y., and M. W. Williams. 1992. Am. J. Physiol. 263:G665-G672). In this study, we have developed an in vivo bile and lymph fistula rat model to test the hypothesis that biliary GSH is an important luminal source of GSH. Peroxidized fish oil was infused into the proximal intestine, and hydroperoxide accumulation in lumen, mucosa, and lymph was determined. Diversion of bile decreased mucosal GSH and increased hydroperoxide accumulation in all fractions. Supplementation with GSH, but not with GSSG, increased tissue GSH and attenuated hydroperoxide accumulation (50-60\%), consistent with enhancement of hydroperoxide removal by exogenous GSH. Addition of native bile deficient in GSH, but not cysteine, cystine, or GSSG, decreased luminal and lymph hydroperoxide levels by 20 $30 \%$. Amino acid supplementation concurrently attenuated hydroperoxide recoveries in these fractions by $30-40 \%$ and increased mucosal GSH by $40 \%$, indicating a role for biliary amino acids in hydroperoxide elimination. The effect of amino acids was abolished by buthionine sulfoximine, confirming their role in GSH biosynthesis. Collectively, the results demonstrate that bile is a rich source of reductant for maintaining mucosal GSH to promote intestinal metabolism of luminal peroxidized lipids. (J. Clin. Invest. 1994. 94:12181225.) Key words: omega-3 fatty acid hydroperoxides • enterohepatic circulation - lymphatic transport of peroxidized lipids • luminal glutathione • biliary amino acids
\end{abstract}

\section{Introduction}

Previous studies have documented that the uptake of exogenous GSH from the intestinal lumen provides an important means for enterocytes to enhance their cellular antioxidant pool (13 ). We recently established that the quantitative removal of

Address correspondence to Dr. Tak Yee Aw, Department of Physiology and Biophysics, Louisiana State University Medical Center, 1501 Kings Highway, Shreveport, LA 71130.

Received for publication 2 September 1993 and in revised form 7 February 1994.

1. Abbreviations used in this paper: $\mathrm{BCNU}, 1,3-\mathrm{bis}$ (2-chloroethyl)1nitrosourea; BF, bile and lymph fistula rat; BSO, L-buthionine- $[S, R]-$ sulfoximine; DHA, docosahexaenoic acid; EPA, eicosapentaenoic acid.

J. Clin. Invest.

(c) The American Society for Clinical Investigation, Inc.

$0021-9738 / 94 / 09 / 1218 / 08 \$ 2.00$

Volume 94, September 1994, 1218-1225 luminal lipid hydroperoxides by rat small intestine is directly related to the mucosal GSH content (4). The enhancement of mucosal GSH levels by exogenous GSH supplementation under conditions in which cellular GSH status is compromised can restore tissue GSH and promote hydroperoxide metabolism (5). Taken together, these findings suggest that a constant supply of luminal GSH would be an important process in the maintenance of normal mucosal GSH. Moreover, the bioavailability of luminal GSH could ultimately determine the efficiency of mucosal hydroperoxide elimination and the susceptibility of the intestinal epithelium to oxidant injury.

Under physiological conditions, luminal GSH can arise from GSH present in the diet $(6,7)$ or from GSH excreted into bile $(8-12)$. An important question that has not been addressed is the functional significance of the enterohepatic circulation in supply of GSH from bile to the small intestine and of the physiological relevance of biliary GSH in intestinal GSH homeostasis and detoxication of luminal oxidants. Despite lingering uncertainties regarding the mechanism and regulation of hepatic GSH transport into bile $(10-14)$, it is now known that the liver puts out as much as $50-60 \%$ of its total GSH export into bile (8, 9). In the rat, minimal autoxidation of GSH occurs during bile transit (15) and, hence, the GSH concentration in rat bile is high (1-2 mM). Thus, this biliary GSH pool can potentially serve as a rich source of reductant for the metabolism of peroxidized lipids by the small intestine. The objective of the current study is, therefore, to test this hypothesis and to define a physiological role of biliary GSH in intestinal hydroperoxide elimination. The approach was to use the conscious bile fistula and lymph fistula rat to quantify luminal and mucosal recoveries and to measure directly lymph output of lipid hydroperoxides after steady state infusions of peroxidized fish-oil solutions. In previous studies, we have established that these parameters are accurate indexes of the efficiency of the metabolism of lipid hydroperoxides by the small intestine $(4,5)$.

\section{Methods}

\section{Animal surgery and postoperative care}

Male Sprague-Dawley rats (250-300 g) were used. Surgery was performed in 24-h fasted rats. In control rats, only the lymph duct was cannulated, whereas in experimental animals (bile fistula [BF] ${ }^{1}$ rats), both the bile and lymph ducts were cannulated. Under isofluorane anesthesia, the major lymph duct above the superior mesenteric artery was cannulated according to Bollman et al. (16). The hepatic bile duct was cannulated with a PE-10 tubing and secured with ties. A duodenal tubing was inserted via the stomach and secured with ties. In some animals, an additional tubing was secured to the wall of the peritoneal cavity for purposes of intraperitoneal administration of GSH-depleting agents. Rats were allowed to recover for $24 \mathrm{~h}$ postoperatively in restraining cages kept in a warm temperature-controlled chamber $\left(30^{\circ} \mathrm{C}\right)$. During this period, the rats were infused with a $5 \%$ glucose-saline solution $(0.3$ M glucose, $145 \mathrm{mM} \mathrm{NaCl}$, and $4 \mathrm{mM} \mathrm{KCl}$ ) at a constant rate of $3 \mathrm{ml} /$ 
$\mathrm{h}$ to replace fluid and electrolyte loss in lymph and bile. The animals were comfortably confined but not tightly restrained under these conditions. All animal procedures have been approved by the Institutional Animal Review Board of the Louisiana State University (LSU) Medical Center.

\section{Preparation of lipid hydroperoxide infusates}

Lipid hydroperoxides were generated by air oxidation of MaxEPA fish oil for $\mathbf{5} \mathrm{d}$. Peroxidized fish-oil emulsions were prepared as previously described $(4,5)$ by sonicating $78 \mu \mathrm{mol}$ egg phosphatidyl choline, 80 $\mu \mathrm{mol}$ MaxEPA fish oil, and $570 \mu \mathrm{mol}$ taurocholate in $30 \mathrm{ml}$ phosphatebuffered saline, $\mathrm{pH}$ 6.4. The amount of lipid hydroperoxides in the emulsions were typically $5 \%$ of the total lipid, and the quantity of lipids received by each rat was standardized to the actual amount of hydroperoxide content ( $1.5 \mu \mathrm{mol})$. GSH, amino acids, and $\gamma$-glutamyl glutamate, whenever present, were added to the lipid emulsions before the infusion at the specified concentrations as follows (mM): $1 \mathrm{GSH}$ 1 each glycine and glutamate, 0.5 cystine, and $10 \gamma$-glutamyl glutamate.

\section{Experimental protocol}

Lipid infusions. Control and BF rats were infused with peroxidized fishoil emulsions without or with GSH (plus or minus $\gamma$-glutamyl glutamate or acivicin) or amino acids at $3 \mathrm{ml} / \mathrm{h}$ for $8 \mathrm{~h}$. Before lipid infusion, some BF animals were pretreated with buthionine sulfoximine (BSO) or with 1,3-bis(2-chloroethyl 1-nitrosourea) (BCNU) as follows: 8 $\mathrm{mmol} / \mathrm{kg}$ BSO in $0.9 \%$ saline was given as a single dose $2 \mathrm{~h}$ before lipid infusion, and $40 \mathrm{mg} / \mathrm{kg} \mathrm{BCNU}$ in $5 \%$ ethanol and diluted with $0.9 \%$ saline was administered $14 \mathrm{~h}$ before lipid infusion. Acivicin $(0.25$ $\mathrm{mM}$ ) was administered at 20 min before lipid infusion and was present throughout the infusion period. Lymph was collected into precooled conical centrifuge tubes at every 2-h interval for $0-2,2-4,4-6$, and 6-8 $\mathrm{h}$ during the 8-h steady state intraduodenal infusion period. All samples were treated with $1 \%$ butylhydrotoluene to prevent oxidation. A $0.5-\mathrm{ml}$ aliquot sample at each time interval was taken for measurements of lipid hydroperoxide. The remainder of the lymph was pooled and extracted with $3 \mathrm{vol}$ of ether for analyses of the fatty acid hydroperoxides by HPLC (see below)

Collection of luminal and tissue samples. Collection was as described previously $(4,5)$. Briefly, at the end of lymph collection, rats were killed by exsanguination under isofluorane anesthesia. The cecum and the small intestine were removed and placed on ice. The small intestine was divided into four equal segments and the luminal contents of each intestinal and cecal segment were thoroughly eluted with three washes of $3 \mathrm{ml}$ of $10 \mathrm{mM}$ sodium taurocholate. The intestinal segments were homogenized, and samples of tissue homogenates and luminal washings (intestinal and cecal) were taken for GSH and lipid hydroperoxide determinations.

\section{Analytical methods}

Total hydroperoxides were determined by the thiobarbituric acid assay as described previously (17). Tissue GSH was determined by HPLC (18). The hydroperoxy eicosapentaenoic (EPA) and docosahexaenoic (DHA) acids, the two major fatty acids of fish oil (19), were analyzed by HPLC as described below. Separation of different lipid fractions in lymph was achieved by TLC (20).

HPLC analyses of fatty acid hydroperoxides. Oxidized triglycerides were digested with pancreatic lipase (21) and purified by TLC (20). The fatty acid hydroperoxide fraction was extracted by ether, dried under nitrogen, and dissolved in a mixture of acetonitrile, water, and tetrahydrofuran (50:40:10, by vol). The different fatty acid hydroperoxides were separated on an ultrasphere reversed-phase octyl $\left(\mathbf{C}_{\mathbf{8}}\right)$ column $(4.6 \times 250 \mathrm{~mm})$ under isocratic conditions on a liquid chromatograph system equipped with model SCL-6B system controller and model LC6A chromatograph (Shimadzu Scientific Instruments, Inc., Houston, TX). General operating conditions were as follows: flow rate, $1 \mathrm{ml} /$ min; running buffer, acetonitrile, water, tetrahydrofuran, and acetic acid $\left(45 \%, 45.8 \%, 9 \%\right.$, and $0.2 \%$, respectively); and temperature, $20^{\circ} \mathrm{C}$.

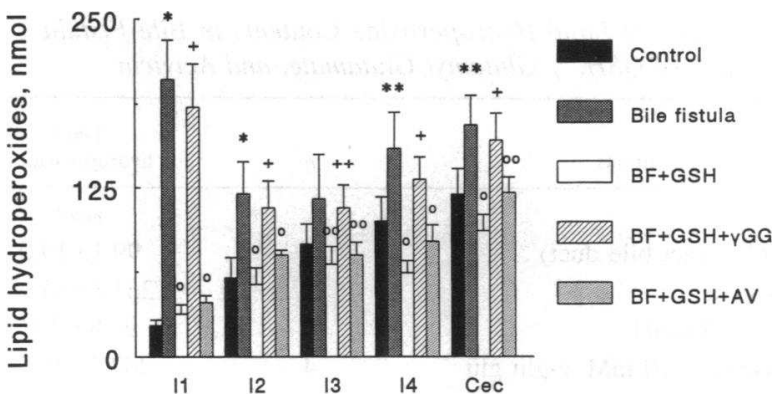

Figure 1. Distribution of luminal lipid hydroperoxides in different segments of the small intestine in BF rats: effect of GSH, $\gamma$-glutamyl glutamate, and acivicin. Lipid emulsions without GSH or with GSH and $\gamma$-glutamyl glutamate or acivicin were infused intraduodenally at $3 \mathrm{ml} / \mathrm{h}$ for $8 \mathrm{~h}$. The small intestine was divided into four equal segments designated as $\mathrm{I}_{1}$ to $\mathrm{I}_{4}$. Lipid hydroperoxides in the luminal contents of the segments and cecum $(\mathrm{Cec})$ were assayed by the thiobarbituric acid method (17). Results are mean \pm SE for intact bile duct control $(n=6$ rats), bile fistula ( $B F ; n=6)$, BF plus $1 \mathrm{mM} \mathrm{GSH}(n=7)$, BF plus GSH and $10 \mathrm{mM} \gamma$-glu glu $(n=4)$, and BF plus GSH and $0.25 \mathrm{mM}$ acivicin $(n=4) .{ }^{*} P<0.001$, and ${ }^{* *} P<0.05$ in comparison with control; ${ }^{\circ} P<0.001$ and ${ }^{\circ} P<0.05$ in comparison with BF; and ${ }^{+} P$ $<0.001$ and ${ }^{++} P<0.01$ in comparison with BF plus GSH. $\gamma-g l u$ glu, $\gamma$-glutamyl glutamate; $A V$, acivicin.

The hydroperoxides were detected at $234 \mathrm{~nm}$ and were quantified by integration of peak areas with respect to standards.

\section{Statistical analysis}

Values are expressed as means \pm SE. Statistical difference between experimental animals and their respective controls were analyzed using one-way analysis of variance.

\section{Materials}

GSH, $\gamma$-glutamyl glutamate, acivicin, L-cystine, L-glutamate, L-glycine, sodium taurocholate, and L-buthionine-[S,R]-sulfoximine (BSO) were purchased from Sigma Chemical Co. (St. Louis, MO). Carmustine (BCNU) was obtained from the Hospital Pharmacy, LSU Medical Center (Shreveport, LA). Egg phosphatidyl choline was obtained from Princeton Lipids (Princeton, NJ). Hydroperoxy fatty acid standards were from Cayman Chemical Co. (Ann Arbor, MI). MaxEPA fish oil was from Seven Seas Health Care Ltd. (Hull, United Kingdom). All other chemicals were of reagent grade and were obtained from local sources.

\section{Results}

Effect of biliary GSH diversion on luminal, tissue, and lymph accumulation of lipid hydroperoxides

Recovery of luminal hydroperoxides. As shown in Fig. 1, the luminal recovery of lipid hydroperoxides was higher in distal than in proximal intestine in control rats (intact bile duct), consistent with a poorer hydroperoxide absorption by the lower bowel $(4,5)$. Bile diversion resulted in significantly higher accumulation of peroxidized lipids in the proximal segments, $I_{1}$ and $I_{2}$, suggesting that intestinal absorption of peroxidized lipids occurs principally in the proximal intestine and that, in the absence of bile, this absorption was markedly impaired.

To evaluate whether the reduction in hydroperoxide absorption was related to the removal of biliary GSH after bile diversion, we supplemented the lipid infusates with $1 \mathrm{mM}$ GSH, a 
Table I. Mucosal Lipid Hydroperoxide Contents In Bile Fistula Rats: Effect of GSH, $\gamma$-Glutamyl Glutamate, and Acivicin

\begin{tabular}{lcc}
\hline \multicolumn{1}{c}{ Conditions } & $n$ & $\begin{array}{c}\text { Total } \\
\text { hydroperoxides }\end{array}$ \\
\hline & & $n m o l$ \\
Control (intact bile duct) & 6 & $99.1 \pm 19.0$ \\
Bile fistula & 6 & $263.8 \pm 33.9 *$ \\
$+1 \mathrm{mM}$ GSH & 7 & $95.8 \pm 7.7^{\ddagger}$ \\
+ GSH + 10 mM $\gamma$-glu glu & 4 & $202.2 \pm 30.7^{8}$ \\
+ GSH + 0.25 mM acivicin & 4 & $93.0 \pm 5.7^{\ddagger}$
\end{tabular}

Values are means \pm SE; $\boldsymbol{n}$, number of rats. Mucosal hydroperoxide levels were determined as the thiobarbituric acid-reactive substances (17) in tissue homogenates. $\gamma$-glu glu, $\gamma$-glutamyl glutamate. ${ }^{*} P<0.001$ in comparison with intact bile duct control. ${ }^{\ddagger} P<0.001$ in comparison with BF. ${ }^{8} P<0.001$ in comparison with BF plus GSH.

concentration that is found in rat bile (15), and measured luminal hydroperoxide recovery. The results show that GSH supplementation significantly attenuated peroxide accumulation in all segments of the intestine (Fig. 1), consistent with a role for GSH in hydroperoxide elimination. To determine if the GSH effect was exerted intracellularly, we inhibited GSH transport by $\gamma$-glutamyl glutamate $(4,5)$. Under these conditions, luminal hydroperoxide accumulation was high, essentially the same as that in the absence of added GSH (Fig. 1), suggesting a requirement for GSH uptake for its effect. In comparison, inhibition of $\gamma$-glutamyl transferase activities by $>95 \%$ with acivicin gave results that were similar to those in the presence of GSH (Fig. 1). This indicates that the mechanism of action of acivicin on $\gamma$-glutamyl transferase differs from that of $\gamma$-glutamyl glutamate under the current conditions and is consistent with $\gamma$ glutamyl glutamate being an inhibitor of intestinal uptake of GSH. Taken together, these results show that biliary GSH is an important exogenous GSH source that can be directly taken up for use by the intestinal mucosa.

Tissue recovery of lipid hydroperoxides. Table I summarizes the results on lipid hydroperoxide recovery in the intestinal mucosa. Tissue hydroperoxide accumulation in BF rats was 2.5fold higher than in control animals, consistent with poor mucosal metabolism of hydroperoxides in bile-diverted animals. This increase in mucosal accumulation was attenuated by exogenous GSH, supporting the view that GSH play an important role in the metabolism of tissue hydroperoxides. The GSH effect was blocked by $\gamma$-glutamyl glutamate but was not affected by acivicin, indicating that mucosal peroxide metabolism was enhanced by GSH transported from the gut lumen. These results are therefore in agreement with the above interpretation that luminal GSH from bile could function to promote intracellular hydroperoxide metabolism.

Lymphatic transport of lipid hydroperoxides. As shown in Fig. 2, lipid hydroperoxide transport into lymph was low under control conditions, similar to previous observations $(4,5)$. In BF rats, the steady state output of hydroperoxide into lymph (4-8 h) was significantly higher than control, consistent with an enhanced hydroperoxide output into lymph consequent to a reduced intracellular metabolism of hydroperoxides in these animals. Interestingly, during the first $2 \mathrm{~h}$ of lipid infusion, the lymphatic transport of peroxidized lipids was low in BF ani-

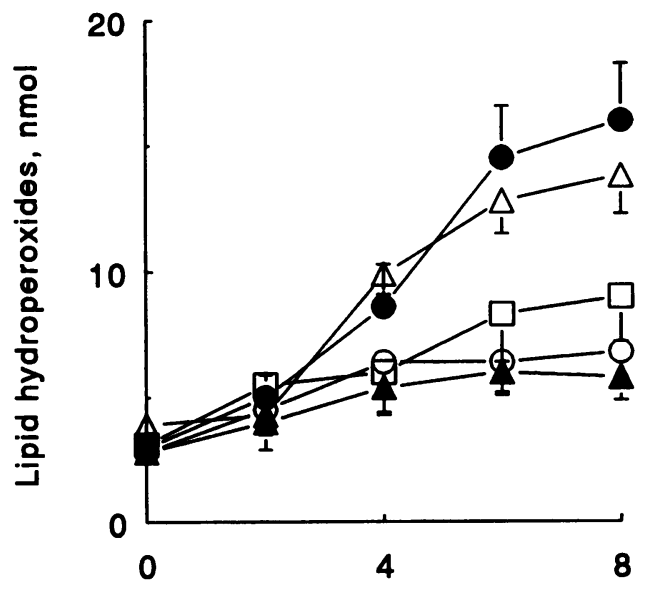

Time of infusion, $h$

Figure 2. Lymphatic transport of lipid hydroperoxides in BF rats: effect of GSH, $\gamma$-glutamyl glutamate, and acivicin. Lipid hydroperoxides were determined in lymph collected from control and experimental rats at 2$h$ intervals over an 8-h steady intraduodenal infusion of peroxidized fish-oil emulsions. Each data point represents mean $\pm S E$ for control ( $n$ $=6$ rats; O), bile fistula $(B F ; n=6 ; \bullet)$, BF plus $1 \mathrm{mM} \mathrm{GSH}(n=7$; $\Delta)$, BF plus GSH and $10 \mathrm{mM} \gamma$-glutamyl glutamate $(n=4 ; \Delta)$, and BF plus GSH plus $0.25 \mathrm{mM}$ acivicin $(n=4 ; \square)$. Differences were observed between the following groups: $\mathrm{BF}$ vs control at 6-8 h ( $P$ $<0.001)$; BF plus GSH vs BF at $4 \mathrm{~h}(P<0.005)$ and at $6-8 \mathrm{~h}(P$ $<0.001$ ); BF plus GSH and $\gamma$-glutamyl glutamate vs BF plus GSH at 4-8 h $(P<0.001)$; BF plus GSH and acivicin vs BF at 6-8 h $(P$ $<0.001)$ and vs BF plus GSH and $\gamma$-glutamyl glutamate at 6-8 h $(P$ $<0.05)$.

mals, which probably reflects the poor absorption of the oxidized lipid (see Fig. 1). GSH supplementation caused a marked reduction in hydroperoxide transport into lymph that was reversed by $\gamma$-glutamyl glutamate (Fig. 2 ). In comparison, supplementation with GSH plus acivicin gave results that were similar to those with GSH alone (Fig. 2). These results collectively show that mucosal uptake of luminal GSH supports tissue hydroperoxide metabolism and attenuates hydroperoxide output into lymph.

Distribution of fatty acid hydroperoxides in lymph lipid fractions. To assess the effects of GSH supplementation on the metabolic fate of EPA and DHA fatty acid hydroperoxides, we analyzed their distribution among the various lipid fractions in lymph. HPLC analyses showed that the oxidation of fish oil yielded equimolar amounts of hydroperoxy fatty acids of EPA and DHA as the major oxidation products (22). As shown in Fig. 3, EPA and DHA hydroperoxides were predominantly associated with triglycerides $(85 \%)$ with a smaller fraction associated with free fatty acids (15\%). In control rats, EPA and DHA hydroperoxide recovery in lymph lipid fractions was low but in BF rats recovery of the two hydroperoxides was significantly increased in the triglyceride fraction with little change in the free fatty acid fraction. This suggests that the oxidized fatty acids are mostly reesterified into triglycerides within the mucosa after their absorption from the lumen. The data in Fig. 3 further revealed that essentially equal amounts of EPA and DHA hydroperoxides were found in lymph triglycerides, indicating that reesterification of the two fatty acid hydroperoxides 


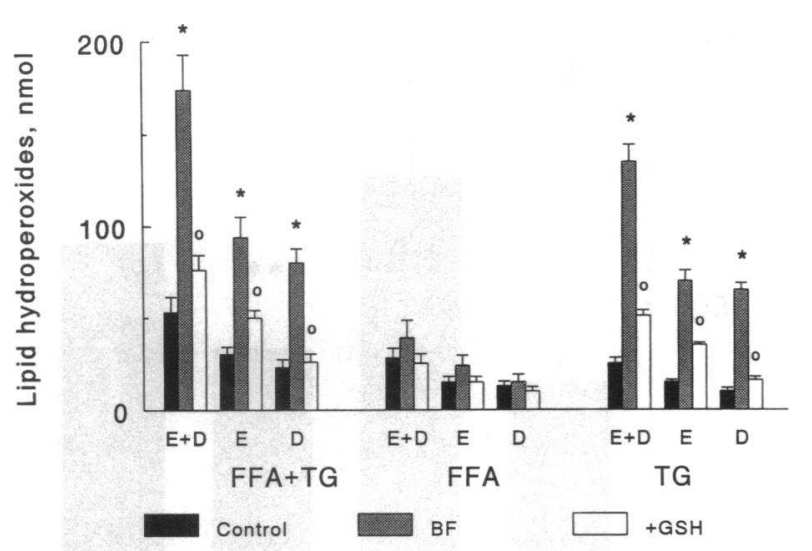

Figure 3. Effect of GSH on distribution of EPA and DHA hydroperoxides in lipid fractions in lymph in BF rats. The triglyceride and free fatty acid fractions in lymph were separated by TLC (19) and the EPA and DHA hydroperoxides in the respective lipid fractions were analyzed by HPLC as described in the text. Results are mean \pm SE for intact bile duct control ( $n=6$ rats), bile fistula $(B F ; n=6)$, and BF plus $1 \mathrm{mM}$ GSH $(n=7) .{ }^{*} P<0.001$ in comparison with control; ${ }^{\circ} P<0.001$ in comparison with $\mathrm{BF}$. $F F A$, free fatty acid; $T G$, triglyceride; $E$, hydroperoxy eicosapentaenoic acid; $D$, hydroperoxy docosahexaenoic acid.

was similar. GSH supplementation markedly reduced EPA and DHA hydroperoxide recovery in lymph triglycerides (Fig. 3), consistent with an enhanced metabolism of the fatty acid hydroperoxides by GSH. The reduction in hydroperoxy DHA was notably greater than the decrease in hydroperoxy EPA, suggesting that DHA hydroperoxides may be preferentially metabolized.

\section{Effect of native bile}

To examine whether other factors in bile besides GSH also influence hydroperoxide elimination, we infused BF rats with peroxidized lipids and native bile that has been essentially depleted of GSH by oxidation (15). The residual concentration of GSH in GSH-deficient bile ranged from 60 to $100 \mu \mathrm{M}$. Under these conditions, the concentrations of cysteine, cystine, and (GSSG) were, respectively, $0.19 \pm 0.03,0.23 \pm 0.05$, and $0.6 \pm 0.09 \mathrm{mM}$. Introduction of GSH-deficient native bile alone caused a $30 \%$ decrease in luminal and a $20 \%$ decrease in lymphatic hydroperoxide levels (Fig. 4). Supplementing native bile with GSH significantly attenuated hydroperoxide recovery in the lumen and lymph by $60 \%$ and $45 \%$, respectively (Fig. 4), similar to the results obtained with GSH alone without bile ( see Figs. 1 and 2). Tissue hydroperoxides were reduced by $30 \%$ with bile supplement compared with BF animals (Table II), whereas the addition of GSH to bile further decreased mucosal hydroperoxide levels (Table II) to near control values. These results show that GSH is a major determinant in the promotion of luminal hydroperoxide removal, but the data also implicate a role for other biliary components in hydroperoxide elimination. Given the relatively high concentrations of cysteine, cystine, and GSSG in native bile (see above), these GSH-related compounds may be important contributors.

\section{Effect of GSH precursor amino acids and GSSG}

To test the above suggestion, BF rats were infused directly with peroxidized fish oil containing GSH precursor amino acids or
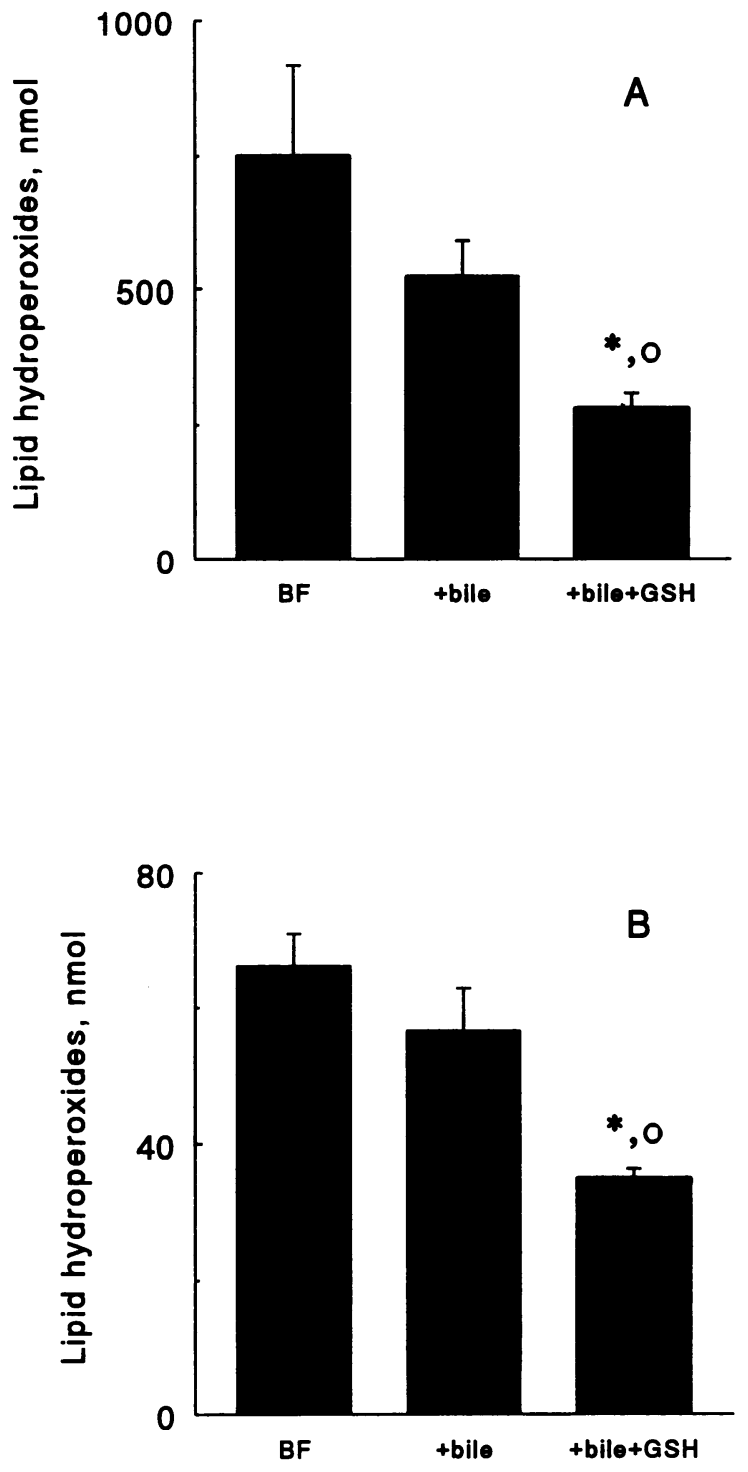

Figure 4. Total luminal and lymph accumulation of lipid hydroperoxides in BF rats: effect of native bile without or with GSH. BF rats were infused intraduodenally with peroxidized fish oil without or with GSHdeficient or GSH-sufficient bile. Luminal and lymph lipid hydroperoxides were determined according to reference 17 . Results are mean \pm SE for $\mathrm{BF}$ rats $(B F ; n=6)$, and $\mathrm{BF}$ rats supplemented with bile $(n=5)$ or with bile and $1 \mathrm{mM} \mathrm{GSH}(n=6) .{ }^{*} P<0.001$ in comparison with $\mathrm{BF} ;{ }^{\circ} P<0.005$ in comparison with $\mathrm{BF}$ plus bile. $(A)$ Luminal hydroperoxides; $(B)$ lymph hydroperoxides.

GSSG. As shown in Fig. 5, supplementation with amino acid mixtures of cystine, glutamate, and glycine caused $40 \%$ decreases in hydroperoxide accumulation in the lumen and lymph and a 50\% reduction in tissue hydroperoxides (Table II). The attenuation of hydroperoxide accumulation by the amino acid mixtures in all fractions was abolished by BSO, an inhibitor of GSH synthesis (Fig. 5, Table II). This indicates that the amino acids function to increase the cellular GSH pool via de novo GSH synthesis. Similar results were obtained when cysteine was used instead of cystine (data not shown), indicating that the intestinal mucosa can readily transport and use both cystine 
Table II. Mucosal Lipid Hydroperoxide Contents In Bile Fistula Rats: Effect of Bile, Amino Acids and GSSG

\begin{tabular}{lcc}
\hline \multicolumn{1}{c}{ Conditions } & $n$ & Total hydroperoxides \\
\hline & & $n$ mol \\
Bile fistula & 5 & $247.9 \pm 36.0$ \\
+ native bile & 5 & $166.7 \pm 12.4^{*}$ \\
+ bile + 1 mM GSH & 6 & $96.3 \pm 5.0^{*}$ \\
+ amino acids & 4 & $114.6 \pm 13.5^{*}$ \\
+ amino acids + BSO & 4 & $247.2 \pm 20.5^{\star}$ \\
+0.5 mM GSSG & 6 & $253.5 \pm 16.5$ \\
\hline
\end{tabular}

Values are means $\pm \mathrm{SE} ; n$, number of rats. Mucosal hydroperoxide contents were determined in tissue homogenates according to reference 17. The amino acid mixtures consisted of $1 \mathrm{mM}$ glycine, $1 \mathrm{mM}$ glutamate, and $0.5 \mathrm{mM}$ cystine. $* P<0.001$ in comparison with $\mathrm{BF}$. ${ }^{\ddagger} P<$ 0.001 in comparison with $\mathrm{BF}$ plus bile. ${ }^{\S} P<0.001$ in comparison with bile plus amino acids.

and cysteine. Overall, these results demonstrate that the enterohepatic recirculation of constituent amino acids may be a physiologically important contributor to mucosal GSH synthesis and to the promotion of intestinal metabolism of fatty acid hydroperoxides.

Interestingly, supplementation of BF rats with GSSG did not affect hydroperoxide accumulation in the intestinal lumen nor the accumulation in lymph, in contrast to a $50-60 \%$ decrease in peroxide recovery with GSH (Fig. 6). GSSG also exerted minimal effects on tissue hydroperoxides (Table II). On the basis of these results, it appears that luminal GSSG is relatively ineffective in promoting hydroperoxide removal as compared with GSH or amino acids. This may be attributed to a lack of reduction of GSSG to GSH in the intestinal lumen under these conditions and/or to a poor cellular uptake and use of GSSG by the intestinal cells.

To compare the effectiveness of GSH and amino acids in the reduction of lipid hydroperoxides, BF animals were infused with different peroxide contents (from 1.0 to $5.0 \mu \mathrm{mol}$ ) and equimolar concentrations of either GSH or the amino acid mixtures under control conditions or under conditions in which animals were pretreated with BCNU to inhibit mucosal GSSG reductase activity ( $55 \%$ inhibition; reference 4 ). In both groups, the rats exhibited marked distension of the small intestine and developed diarrhea at peroxide concentrations $>2 \mu \mathrm{mol}$. In addition, between 4 and $8 \mathrm{~h}$ of the lymph collection period, lymph flow in control and experimental BF animals fell from 3 to $0.8 \mathrm{ml} / \mathrm{h}$, indicating loss of absorptive and lymphatic functions. At the peroxide dose used in the current study $(1.5 \mu \mathrm{mol})$, GSH and amino acids appear to exert similar effects on the accumulation of luminal and lymph lipid hydroperoxides (Figs. 5 and 6 ). These results are consistent with an inability of animals with severely compromised detoxication capacity to handle high peroxide doses efficiently; as a consequence, excessive hydroperoxide could accumulate in the intestinal lumen and lead to mucosal fluid loss and diarrhea.

\section{Tissue levels of GSH}

To verify that the changes in luminal, mucosal, and lymph hydroperoxide contents related to bile diversion and of GSH and amino acid supplementation were associated with altered
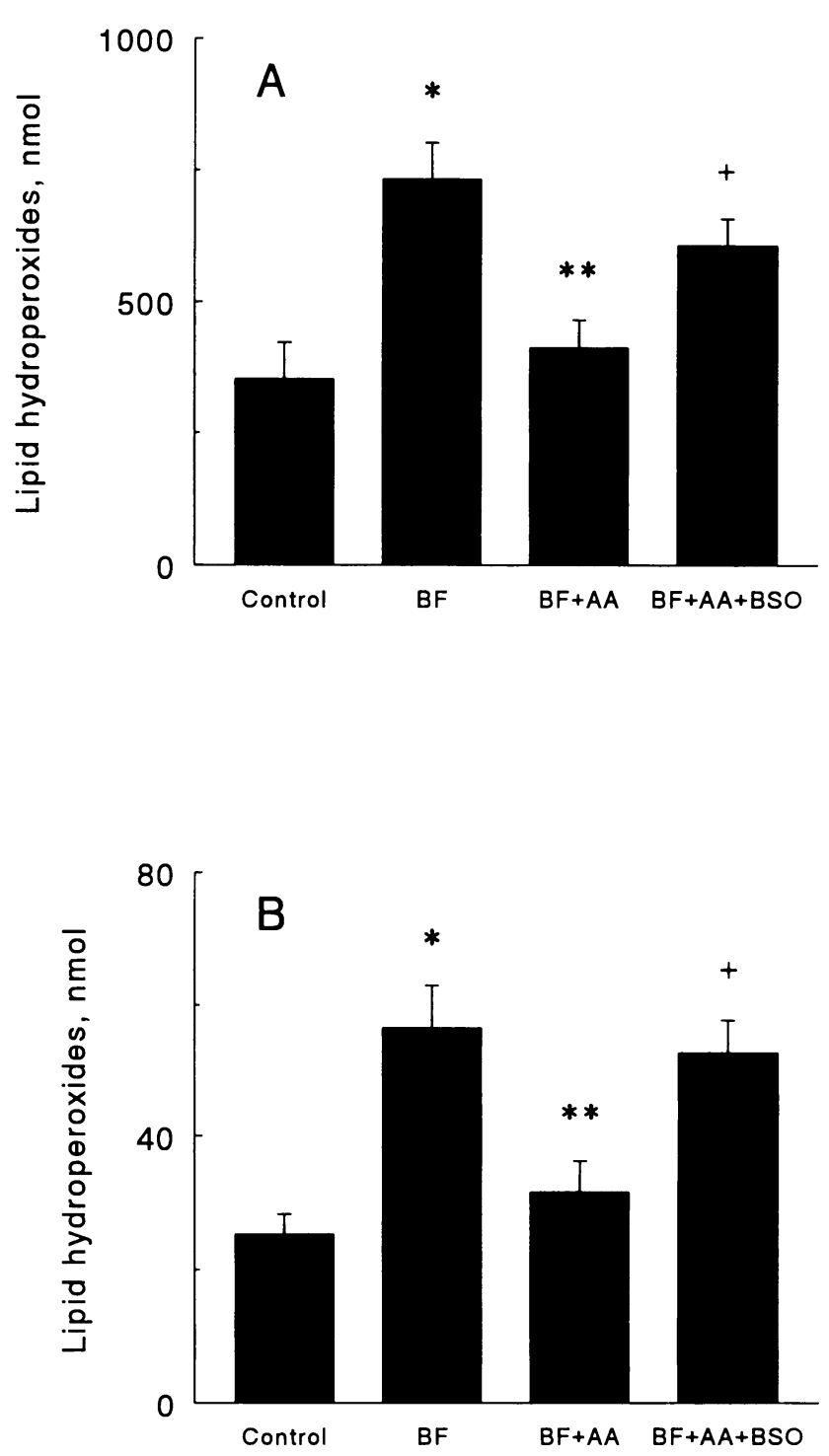

Figure 5. Effect of amino acids on total luminal and lymph accumulation of lipid hydroperoxides in BF rats. Luminal and lymph lipid hydroperoxides were determined in BF rats infused with peroxidized lipids without or with amino acid supplements. Results are mean $\pm \mathrm{SE}$ for control (intact bile duct; $n=5$ ), BF rats ( $B F ; n=6$ ), and BF plus amino acids $(n=4)$ or BF pretreated with BSO and supplemented with amino acids $(n=4), * P<0.001$ in comparison control; $* * P$ $<0.001$ in comparison with $\mathrm{BF}$ : ${ }^{+} P<0.005$ in comparison with $\mathrm{BF}$ plus AA. $A A$, amino acid; $B S O$, buthionine sulfoximine. ( $A$ ) Luminal hydroperoxides; $(B)$ lymph hydroperoxides.

mucosal GSH, we measured tissue GSH concentrations. Samples of the intestinal mucosa were obtained at the end of lipid infusion, and GSH was determined by HPLC (18) in the trichloroacetic acid extracts of tissue homogenates. Basal mucosal GSH levels in rats not exposed to lipids were $2.71 \pm 0.27 \mu \mathrm{mol} /$ $\mathrm{g}$ tissue and were not significantly altered after lipid infusion (2.63 $\pm 0.35 \mu \mathrm{mol} / \mathrm{g}$; Table III). Tissue GSSG contents also remained unchanged $(0.093 \pm 0.010 \mathrm{vs} 0.10 \pm 0.005 \mu \mathrm{mol} / \mathrm{g}$ for control and lipid-supplemented animals, respectively). Intestinal GSH contents in BF rats with lipid exposure were $1.47 \pm 0.11$ 

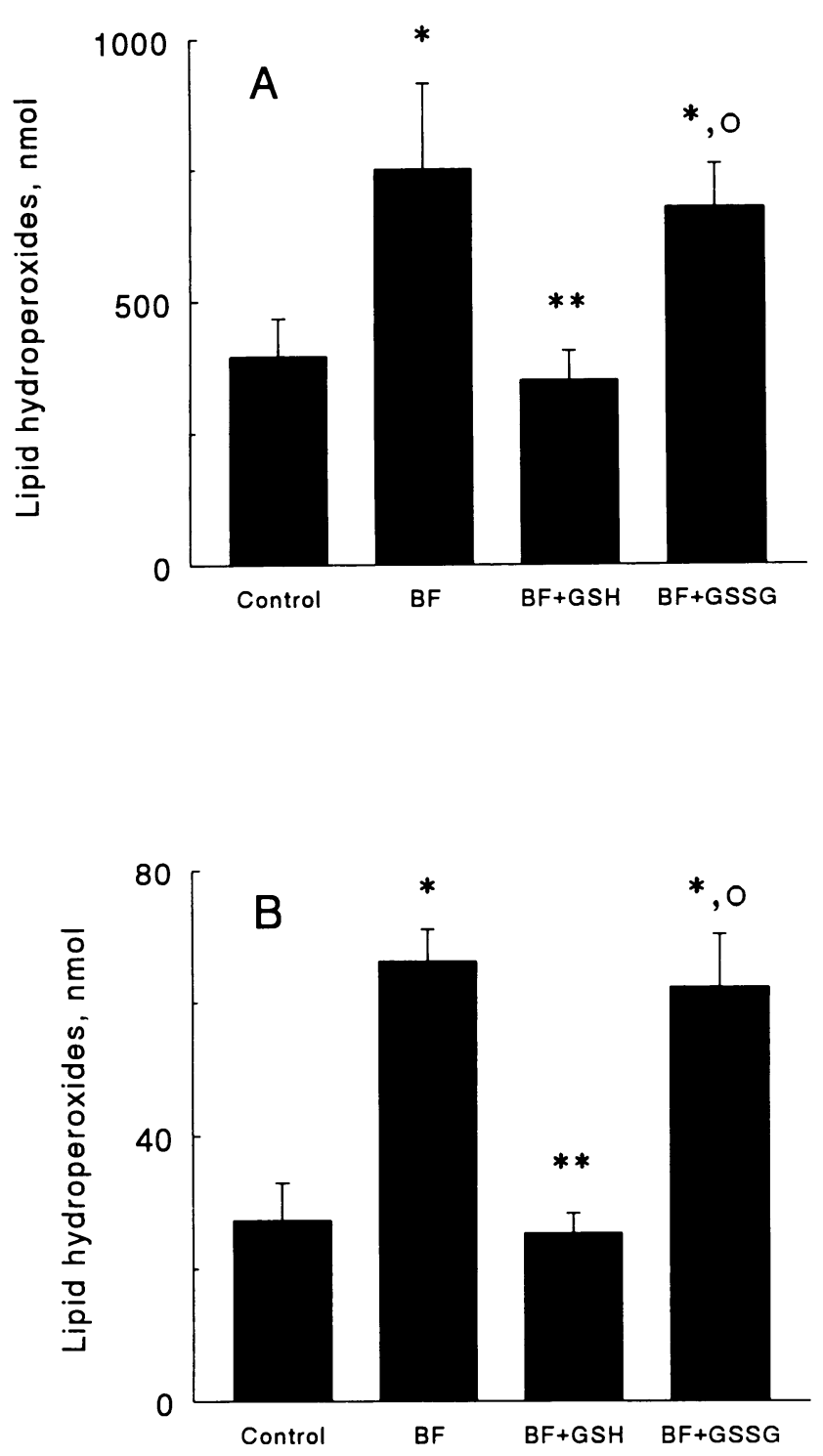

Figure 6. Effect of GSSG on total luminal and lymph accumulation of lipid hydroperoxides in BF rats. BF rats were infused with peroxidized fish oil and GSH or GSSG, and the total luminal and lymph lipid hydroperoxide contents were determined as described in the text. Results are mean $\pm \mathrm{SE}$ for control (intact bile duct; $n=6$ ), BF rats ( $B F ; n$ $=7)$, BF plus GSH $(n=5)$, or BF plus GSSG $(n=6) . * P<0.001$ in comparison with control: $* * P<0.001$ in comparison with $\mathrm{BF}:{ }^{\circ} P$ $<0.005$ in comparison with BF plus GSH. $(A)$ Luminal hydroperoxides; (B) lymph hydroperoxides.

$\mu \mathrm{mol} / \mathrm{g}$; Table III) and were not significantly different from the values in animals without peroxide exposure $(1.51 \pm 0.09$ $\mu \mathrm{mol} / \mathrm{g}$ ). These results show that acute exposure of the mucosa to a low dose of hydroperoxide caused little change in tissue GSH levels, suggesting that during low oxidant stress. steady state GSH status was well maintained.

Mucosal GSH concentrations were 50\% lower in BF rats than in rats with intact bile duct (Table III), supporting a role for biliary GSH in the maintenance of normal tissue GSH levels. Exogenous GSH supplementation in BF animals increased mucosal GSH concentrations to above control levels (Table III),
Table III. Mucosal GSH Concentrations in Bile Fistula Rats Under Control and Different Experimental Conditions

\begin{tabular}{|c|c|c|}
\hline Conditions & $n$ & GSH \\
\hline & & $\mu \mathrm{mol} / \mathrm{g}$ tissue \\
\hline Control (intact bile duct) & 6 & $2.63 \pm 0.35$ \\
\hline Bile fistula & 6 & $1.47 \pm 0.11 *$ \\
\hline$+1 \mathrm{mM} \mathrm{GSH}$ & 7 & $3.34 \pm 0.24$ \\
\hline$+\mathrm{GSH}+10 \mathrm{mM} \gamma$-glu glu & 4 & $1.66 \pm 0.26^{* 8}$ \\
\hline$+\mathrm{GSH}+0.25 \mathrm{mM}$ acivicin & 4 & $3.15 \pm 0.35$ \\
\hline+ native bile & 5 & $1.77 \pm 0.18 *$ \\
\hline+ bile $+1 \mathrm{mM} \mathrm{GSH}$ & 6 & $3.56 \pm 0.32$ \\
\hline+ amino acids & 4 & $2.44 \pm 0.18$ \\
\hline+ amino acids + BSO & 4 & $1.58 \pm 0.09 *$ \\
\hline$+0.5 \mathrm{mM}$ GSSG & 6 & $1.59 \pm 0.11^{*}$ \\
\hline
\end{tabular}

Values are means \pm SE; $n$, number of rats. Intestinal GSH concentrations were determined in trichloroacetic acid-soluble supernatants of tissue homogenates by HPLC (18). The amino acid mixtures consisted of 1 $\mathrm{mM}$ glycine. $1 \mathrm{mM}$ glutamate, and $0.5 \mathrm{mM}$ cystine. $\gamma$-glu glu, $\gamma$ glutamyl glutamate. $* P<0.001$ in comparison with intact bile duct control. ${ }^{\ddagger} P<0.001$ in comparison with $\mathrm{BF}$. ${ }^{\star} P<0.001$ in comparison with BF plus GSH. $P<0.005$ in comparison with $\mathrm{BF}$ plus amino acids.

suggesting that biliary GSH can enhance the intracellular GSH pool. The increase in GSH levels associated with GSH supplementation was ameliorated by $\gamma$-glutamyl glutamate but was not affected by acivicin (Table III), consistent with GSH transport by intestinal cells. The addition of GSH-depleted native bile increased tissue GSH by $20 \%$, whereas inclusion of GSH elevated cellular GSH to above control levels (Table III). Supplementation with amino acids also elevated mucosal GSH levels, which was prevented by BSO (Table III), confirming the role of amino acids in GSH synthesis. Exogenous GSSG was without effect on tissue GSH, which supports the above suggestion that GSSG was not a major source of cellular GSH under these conditions. Taken together, these results are consistent with biliary GSH and amino acids being important luminal sources of reductant, either via direct uptake or via biosynthesis, respectively, for the maintenance of the intracellular GSH concentrations.

\section{Discussion}

In the current study, the use of a bile and lymph fistula rat model provides a suitable in vivo approach to evaluate the physiological relevance of biliary GSH in intestinal handling of peroxidized lipids. This experimental approach was based on our earlier studies with the conscious lymph fistula rat in which we found a direct quantitative relationship between the intestinal elimination of luminal lipid hydroperoxides and the mucosal GSH status (4). We further found that exogenous GSH supplementation was effective in restoring tissue GSH and promoting hydroperoxide removal from the gut lumen under conditions of GSH deficiency (5).

The current studies show that the absence of luminal bile caused an overall decrease in the metabolism of peroxidized lipids by the small intestine as reflected in significant accumula- 
tion of lipid hydroperoxides in the intestinal lumen and mucosa and enhanced output of hydroperoxides into lymph. This marked impairment in hydroperoxide metabolism in the absence of bile was associated with decreases in mucosal GSH concentrations that was mostly attributable to the diversion of the biliary GSH pool. Consequently, restoration of this GSH pool by exogenous GSH supplementation concurrently promoted lipid hydroperoxide elimination from the lumen and attenuated lymphatic hydroperoxide output. These results therefore support a physiological role for biliary GSH in intestinal metabolism of lipid peroxides. More importantly, the study provides the first evidence for the importance of the function of the enterohepatic circulation of GSH in the detoxication of luminal oxidants, such as peroxidized lipids.

The dependence of hydroperoxide metabolism on the quantitative status of mucosal GSH suggests that a high cell GSH is required to support GSH peroxidase function during enhanced hydroperoxide reduction (23). In support of a role of the GSH redox cycle in the detoxication of fatty acid hydroperoxides, we found that hydroperoxy EPA and DHA are excellent substrates for the soluble seleno GSH peroxidase (Aw, T. Y., and Williams, M. W., unpublished results). In comparison, more hydrophobic lipid hydroperoxides, such as hydroperoxycholesterol, are predominantly metabolized by the membrane-associated phospholipid peroxidase (24), suggesting that the relative water solubility of peroxidized lipids could determine their cellular pathway of metabolism.

A reasonable interpretation of the function of GSH in the removal of luminal peroxidized lipids is intracellular metabolic trapping. The removal of products during high hydroperoxide metabolism under GSH-sufficient status is expected to create a large extracellular-to-intracellular hydroperoxide gradient for greater uptake from the lumen. In contrast, at low tissue GSH, luminal hydroperoxide uptake may be rate limited by decreased intracellular hydroperoxide metabolism due to saturation of the compromised GSH peroxidase system. Other lines of evidence are consistent with hydroperoxide metabolism being mostly an intracellular event. Kowalski et al. (25) reported the absence of GSH peroxidase at intestinal brush border membranes and we found negligible luminal enzyme activities (Aw, T. Y., unpublished results). Hence, it is unlikely that hydroperoxides are substantially metabolized at the intestinal cell membranes or within the intestinal lumen. Our finding that inhibition of GSH transport prevented the exogenous effect of GSH on hydroperoxide accumulation further attests to an intracellular mode of hydroperoxide metabolism by GSH-dependent mechanisms. Although our previous $(4,5)$ and current data is consistent with this interpretation, we cannot, at present, rule out other explanations of the results, such as direct inhibition of hydroperoxide uptake under GSH-deficient conditions or the facilitative access of hydroperoxides to the cell membrane consequent to the mucolytic effect of luminal GSH on the mucus layer.

The finding that hydroperoxide levels were affected markedly despite saturation levels of the remaining tissue GSH may best be explained by the fact that we have considered mucosal GSH as a single homogenous pool. This oversimplified presentation provides little specific information on hydroperoxide-induced depletion of GSH in different regions of the intestinal villous. To address this problem, we attempted to separate tissue GSH pools into villous tip, mid-villus, and crypt by differential scraping of the mucosal layer. Unfortunately, the various layers were not cleanly separated by this technique, and the results obtained were ambiguous and unreproducible. Alternatively, we have isolated different enterocyte cell populations along the villi using the fractionation procedure of Weiser (26). However, given the length of time needed for differential cell isolation $(3 \mathrm{~h})$ and the time taken for lymph collection $(8 \mathrm{~h})$ for each animal, it was technically not feasible to combine the two methods in the same experiment. Thus, we cannot, at present, fully address the question of changes in hydroperoxide contents with regional changes in tissue GSH.

It is significant that GSH precursor amino acids are effective in enhancing intestinal mucosal GSH and promoting hydroperoxide elimination since biliary ductular epithelium $(27,28)$ and the intestinal brush border (29) is rich in $\gamma$-glutamyl transferase, which functions to degrade GSH (30), and luminal uptake of intact GSH occurs concurrently with hydrolysis of GSH, uptake of the constituent amino acids $(2,31)$, and intracellular GSH resynthesis. The current observation that luminal amino acids can be efficiently taken up by the intestine for intracellular GSH synthesis suggests that the recirculation of biliary GSH precursor amino acid could provide an important physiological mechanism to bolster intestinal antioxidant defenses, such as in human small intestine, given the low GSH content in human bile (Aw, T. Y., unpublished results).

A recognized limitation of the current in vivo approach is the lack of precise quantification of the respective contribution of GSH and amino acids to the maintenance of the steady state cellular GSH pool. Additionally, the source of reductant supply, i.e., luminal versus plasma, also cannot be readily assessed using this approach. Notwithstanding, recent studies provide some insights into the quantitative contribution of GSH synthesis and GSH uptake to maintaining enterocyte GSH concentration. We found that the rate of GSH synthesis in freshly isolated enterocytes from rat proximal intestine was low $(0.031 \pm 0.002$ $\mathrm{nmol} / 10^{6}$ cells per minute; reference 32 ) relative to other organs such as the liver $\left(0.4-0.8 \mathrm{nmol} / 10^{6}\right.$ cells per minute; reference 33). By comparison, the rate of GSH uptake by enterocytes from the extracellular medium was $0.077 \pm 0.003$ $\mathrm{nmol} / 10^{6}$ cells per minute (32). Based on these studies in isolated enterocytes, the rate of GSH transport is at least twofold greater than the rate of GSH synthesis. If similar rates occur in vivo, the uptake of GSH from the intestinal lumen may quantitatively be more important than GSH synthesis in the optimal supply of GSH during enhanced hydroperoxide metabolism.

Since phospholipids and bile salts are requisites for normal absorption of nonoxidized lipids (34), biliary amphiphilic compounds may also play a role in the absorption of oxidized fats. At present, little is known about the physicochemical properties of peroxidized lipids in the intestinal lumen and how oxidative modification of lipids could alter their physicochemical behavior in the luminal phases and formation of mixed micelles. Also, little is known of the cellular processing of fatty acid hydroperoxides after absorption. Our study shows that oxidized long-chain fatty acids are substrates for reesterification as evidenced by the significant association of hydroperoxy fatty acids with lymph triglycerides. Not surprisingly, however, the intestinal absorption and reesterification of oxidized long-chain unsaturated fatty acids are substantially poorer in comparison to those of nonoxidized unsaturated fatty acids (35). Collectively, the current results underscore the significant impact that oxidation 
has on the absorptive, metabolic, and lymphatic transport phases of luminal lipids.

In summary, we have established an in vivo bile and lymph fistula rat model to evaluate the physiological significance of bile in intestinal metabolism of luminal peroxidized lipids. The results provided evidence for the importance of biliary GSH and its amino acids in maintaining mucosal GSH and enhancing cellular metabolism of lipid hydroperoxides. Since high lipid intake can promote oxidative injury to the intestinal epithelium by increased lipid peroxidation and subsequent propagation of oxygen radicals $(36,37)$, the promotion of GSH-dependent hydroperoxide detoxication can effectively decrease the risk for development of various gut pathologies, such as inflammation and cancer.

\section{Acknowledgments}

I thank Dollie F. Smith and Alan D. Williams for excellent technical assistance.

This study was supported by the National Institute of Diabetes and Digestive Kidney Diseases grant DK-44510. T. Y. Aw is a recipient of the American Heart Association Established Investigatorship Award.

\section{References}

1. Hagen, T. M., and D. P. Jones. 1987. Transepithelial transport of glutathione in vascularly perfused small intestine of rat. Am. J. Physiol. 252:G607-G613.

2. Hagen, T. M., G. T. Wierzbicka, B. B. Bowman, T. Y. Aw, and D. P. Jones. 1990. Fate of dietary glutathione: disposition in the gastrointestinal tract. Am. J. Physiol. 259:G530-G535.

3. Martensson, J., A. Jain, and A. Meister. 1990. Glutathione is required for intestinal function. Proc. Natl. Acad. Sci. USA. 87:1715-1719.

4. Aw, T. Y., M. W. Williams, and L. Gray. 1992. Absorption and lymphatic transport of peroxidized lipids by rat small intestine in vivo: role of mucosal GSH. Am. J. Physiol. 262:G99-G106.

5. Aw, T. Y., and M. W. Williams. 1992. Intestinal absorption and lymphatic transport of peroxidized lipids in rats: effect of exogenous GSH. Am. J. Physiol. 263:G665-G672.

6. Jones, D. P., R. J. Coates, E. W. Flagg, J. W. Eley, G. Block, R. S Greenberg, E. W. Gunter, and B. Jackson. 1992. Glutathione in foods listed in the National Cancer Institute's Health Habits and History Food Frequency Questionaire. Nutr. Cancer. 17:57-75.

7. Samiec, P. S., L. J. Dahm, E. W. Flagg, R. J. Coates, J. W. Eley, and D. P. Jones. 1993. Bioavailability of dietary glutathione. In Free Radical and Antioxidants in Nutrition. F. Corongiu, S. Banni, M. A. Desi, and C. Rice-Evans, editors. Richelieu Press, London. 269-285.

8. Ballatori, N., A. T. Truong, A. K. Ma, and J. L. Boyer. 1989. Determinants of glutathione efflux and biliary GSH/GSSG ratio in perfused rat liver. Am. J. Physiol. 256:G483-G490.

9. Ookhtens, M., and T. Maddatu. 1991. Mechanism of changes in hepatic sinusoidal and biliary glutathione efflux with age in the rat. Am. J. Physiol. 261:G648-G656.

10. Kaplowitz, N., D. E. Eberle, J. Petrini, J. Touloukian, M. C. Corvasce, and F. Kuhlenkamp. 1983. Factors influencing the efflux of hepatic glutathione into bile in rats. J. Pharmacol. Exp. Ther. 224:141-147.

11. Lauterberg, B. H., C. V. Smith, H. Hughes, and J. R. Mitchell. 1984 Biliary excretion of glutathione and glutathione disulfide in the rat. Regulation and response to oxidative stress. J. Clin. Invest. 73:124-133.

12. Ballatori, N., and T. W. Clarkson. 1985. Sulfobromophthalein inhibition of glutathione and methylmercury secretion into bile. Am. J. Physiol. 248:G238G245.
13. Inoue, M., R. Kinne, T. Tran, and I. M. Arias. 1983. The mechanism of biliary secretion of reduced glutathione. Analysis of transport process in isolated rat liver canalicular membrane vesicles. Eur. J. Biochem. 134:467-471.

14. Fernandez-Checa, J. C., H. Takikawa, T. Horie, M. Ookhtens, and N. Kaplowitz. 1992. Cannalicular transport of reduced glutathione in normal and mutant Eisai hyperbilirubinemic rats. J. Biol. Chem. 267:1667-1673.

15. Eberle, D., R. Clarke, and N. Kaplowitz. 1981. Rapid oxidation in vitro of endogenous and exogenous glutathione in bile of rats. J. Biol. Chem. 256:21152117.

16. Bollman, J. L., J. C. Cain, and J. H. Grindlay. 1949. Techniques for the collection of lymph from the liver, small intestine, or thoracic duct of the rat. $J$. Lab. Clin. Med. 33:1329-1352.

17. Buege, J. A., and S. D. Aust. 1978. Microsomal lipid peroxidation. Methods Enzymol. 52:302-310.

18. Reed, D. J., J. R. Babson, P. W. Beatty, A. E. Brodie, W. W. Ellis, and D. W. Potter. 1980. High-performance liquid chromatography analysis of nanomole levels of glutathione, glutathione disulfide, and related thiols and disulfides. Anal. Biochem. 106:55-62.

19. Gunstone, F. D., J. L. Harwood, and F. B. Padley. 1986. The Lipid Handbook. Chapman and Hall, London. 314 pp.

20. Funk, M. O., R. Issac, and N. A. Porter. 1975. Preparation and purification of lipid hydroperoxides from arachidonic and $\gamma$-linolenic acids. Lipids. 11:113117.

21. Aw, T. Y., and M. R. Grigor. 1978. Effect of intramolecular fatty acid distribution on aspects of triacylglycerol digestion and absorption studied in vitro. Biochem. Biophys. Acta. 531:257-265.

22. Cepinskas, G., P. R. Kvietys, and T. Y. Aw. 1994. Omega 3 lipid peroxides injure CaCo-2 cells: relationship to development of GSH antioxidant systems. Gastroenterology. 107:80-86.

23. Chance, B., H. Sies, and A. Boveris. 1979. Hydroperoxide metabolism in mammalian organs. Physiol. Rev. 59:527-605.

24. Geiger, P. G., J. P. Thomas, and A. W. Girotti. 1991. Lethal damage to murine L1210 cells by exogenous lipid hydroperoxides. Protective role of glutathione-dependent selenoperoxidases. Arch. Biochem. Biophys. 288:671-680.

25. Kowalski, D. P., R. M. Feeley, and D. P. Jones. 1990. Use of exogenous glutathione for metabolism of peroxidized methyl linoleate in rat small intestine. J. Nutr. 120:1115-1121.

26. Weiser, M. M. 1973. Intestinal epithelial cell surface membrane glycoprotein synthesis. I. An indicator of cellular differentiation. J. Biol. Chem. 248:25362541 .

27. Abbott, W. A., and A. Meister. 1986. Intrahepatic transport and utilization of biliary glutathione and its metabolism. Proc. Natl. Acad. Sci. USA. 83:12461250.

28. Ballatori, N., R. Jacob, and J. L. Boyer. 1986. Intrabiliary glutathione hydrolysis: a source of glutamate in bile. J. Biol. Chem. 261:7860-7865.

29. Szewczuk, A., H. Milenrowitz, M. V. Polosatov, and K. A. Sobeich. 1980 Immunofluorescent localization of g-glutamyl transferase in rat and bovine tissues. Acta Histochem. 66:152-159.

30. Glenner, G. G., and J. E. Folk. 1961. Glutamyl peptidases in rat and guinea pig kidney slices. Nature (Lond.). 192:338-340.

31. Hagen, T. M., G. T. Wierzbicka, A. H. Sillau, B. B. Bowman, and D. P. Jones. 1990. Bioavailability of dietary glutathione: effect on plasma concentration. Am. J. Physiol. 259:G524-G529.

32. Williams, A. D., and T. Y. Aw. 1994. Glutathione (GSH) supply for lipid hydroperoxide metabolism in intestinal epithelial cells. Gastroenterology. (Abstr.) 106:1054.

33. Shan, X., T. Y. Aw, R. Shapira, and D. P. Jones. 1989. Oxygen dependence of glutathione synthesis in hepatocytes. Toxicol. Appl. Pharmacol. 101:261-270.

34. Tso, P., H. Kendrick, J. A. Balint, and W. J. Simmonds. 1981. Role of biliary phosphatidylcholine in the absorption and transport of dietary triolein in the rat. Gastroenterology. 80:60-65.

35. Bergstedt, S. E., H. Hayashi, D. Kritchevsky, and P. Tso. 1990. A comparison of absorption of glycerol tristearate and glycerol trioleate by rat small intestine. Am. J. Physiol. 259:G386-G393.

36. Parks, D. A., G. B. Bulkley, and D. N. Granger. 1983. Role of oxygenderived free radicals in digestion tract diseases. Surgery. 94:415-422.

37. Grisham, M. B., and D. N. Granger. 1988. Neutrophil-mediated mucosal injury. Role of reactive oxygen metabolites. Dig. Dis. Sci. 33:6S-15S. 